\title{
Encoding-retrieval interactions in memory for implicational sentences
}

\author{
ROBERT E. TILL \\ University of North Dakota, Grand Forks, North Dakota
}

\begin{abstract}
In two experiments, subjects listened to a list of implicational sentences and performed either a comprehension or a memorization task. They were subsequently given a test of free recall or cued recall (with implications as cues). Performance in the comprehension/cued-recall condition was consistently better than that in the dissimilar-encoding/retrieval conditions (memorization/cued recall and comprehension/free recall), supporting the hypothesis that encoding/retrieval similarity facilitates recall. In contrast, performance in the memorization/free-recall condition was not consistently better than in the dissimilar-encoding/retrieval conditions. Thus, the advantage of encoding/retrieval similarity for recall was clear only in the case of distinctive encoding (specific comprehension responses) and distinctive retrieval cues (implications as cues). The results show the relevance of distinctiveness and encoding/retrieval compatibility for memory of meaningful material.
\end{abstract}

Several researchers have argued that semantic tasks do not provide inherently stronger memory traces than do nonsemantic tasks (e.g., Jenkins, 1979; Nelson, 1979; Tulving, 1979). Tulving (1979), in particular, suggested that the levels-of-processing notion adds nothing beyond that already contained in the "encoding specificity principle." $\mathrm{He}$ emphasized the interactive effect of encoding and retrieval conditions on memory performance. Some kinds of encoding lead to good performance under some conditions of retrieval. Similarly, Morris, Bransford, and Franks(1979) referred to "transfer appropriate processing" and suggested that the value of a particular kind of encoding depends on the goal of the learning experience. Tulving (1979) discussed the notion of "optimal" encoding/retrieval similarity, but warned against the tendency to equate optimal similarity with nominal identity. Both he and Craik (1979) considered the question of why encoding/retrieval similarity leads to performance that is higher in some cases than in others, and both suggested that future work should examine the uniqueness or distinctiveness of traces.

The present study examined the role of encoding/ retrieval similarity in memory for implicational sentences. While much of the work supporting the memory advantage in cases of encoding/retrieval similarity has used word lists, several sentence memory studies have also been concerned with the encoding/retrieval relationship. Most of these have manipulated encoding by varying the verbal context or sentence in which the stimulus (phrase or sentence) appears (e.g., Anderson \& Ortony, 1975; Corbett \& Dosher, 1978; Till, 1977). There has been little attempt to vary the study instruc-

The author's mailing address is: Robert E. Till, Psychology Department, Box 7187, University Station, Grand Forks, North Dakota 58202. tions given to subjects. Some subjects have been instructed to try to understand each sentence (e.g., Till, 1977), whereas others have been told to read and remember the sentences (e.g., Corbett \& Dosher, 1978). It is not known to what extent such study factors add to the text variables affecting encoding.

The present study was designed as a naturalistic demonstration of encoding/retrieval similarity in sentence memory. All subjects listened to the same sentences, each with a high-probability implication. The subjects performed either a comprehension task or a memorization task. Both were described as familiar, meaningful tasks, although the comprehension task led to a specific, unique response to each sentence, whereas the memorization task did not. Subjects were tested for cued recall or free recall of the sentences. In the cued-recall condition, implicational cue words appeared on the test page.

It was expected that encoding/retrieval similarity would be greatest for the memorization/free-recall and comprehension/cued-recall conditions. Encoding and retrieval activities here were thought to be well matched. Thus, recall should be highest in these two conditions. Furthermore, if, in addition to encoding/retrieval similarity, uniqueness or distinctiveness of the memory trace or cue is important, then the comprehension/ cued-recall condition should lead to better recall than the memorization/free-recall condition. Experiment 1 tested the basic predictions of this $2 \times 2$ design. Experiment 2 added a presentation rate variable to the design.

\section{EXPERIMENT 1}

\section{Method}

Subjects. All subjects were undergraduates from introductory psychology courses at Southern Methodist University in Dallas. Each received course credit for participation. The subjects were 
tested in small groups assigned randomly to either free recall or cued recall. Within each group, some subjects were assigned the memorization task and others were assigned the comprehension task. There were 80 subjects in the $2 \times 2$ design.

Materials. A list of stimulus sentences was constructed by selecting 16 sentences from the Till, Cormak, and Prince (1977) study. The sentences were worded such that certain implications would have a high probability, at least for subjects engaged in a comprehension task. The following examples were used in the experiment: (1) "The hostess raised her glass in the air," and (2) "The pupil carefully positioned the thumbtack on the chair." The sentences varied in length from 5 to 10 words. A single randomized order of presentation was used for all subjects.

A list of 16 recall cues, one for each sentence, was also prepared. Each cue was a noun referring to information that might be inferred from the sentence, such as an object involved in the described event or the social significance of the event. The following cues correspond to the sentences given above: (1) "toast," and (2) "prank." The subjects in the cued-recall condition were given a page with the cue words listed on it. One order of cue words was used.

Procedure. In each session, the subjects were arbitrarily assigned either the memorization task or the comprehension task. Directions for the task were printed on a page, and the subjects were given time to read them. The directions were repetitious but can be summarized as follows:

In the comprehension task, the subjects were told to think about the event described in each sentence and the things that might be involved in this event. Then they were to write down a word that reflected their understanding of the sentence. Examples were provided. In the memorization task, the subjects were instructed to listen to each sentence and to try to learn it. These subjects were not allowed to write anything down.

All subjects were told that there would be more to the experiment, but were given no details about recall testing. The experimenter read each stimulus sentence at the rate of one new sentence every $20 \mathrm{sec}$. Each sentence was read and immediately repeated to minimize errors of speech perception. Each subject then performed the assigned task.

After presentation of the sentences, the subjects were given recall instructions. Thus, approximately $1 \mathrm{~min}$ after the sentence input list, the subjects were given an answer page for recall. Those in the free-recall condition received a blank page, and those in cued recall received a page with the 16 cue words. Free-recall subjects were instructed to write down sentences they remembered in any order they wished. In cued recall, the subjects were told that each cue related to a sentence presented earlier and that some of the cues would be more effective than others. Responses were to be written alongside the relevant cue. All subjects were told to use the exact wording when possible, but they were told that guesses, omissions, and repetitions were permissible. Four minutes were allowed for recall.

Scoring. The subjects' responses were scored according to the lenient criteria used by Till et al. (1977). Sentences recalled verbatim as well as sentences containing some substitutions or omissions were considered acceptable. For example, sentences with omissions of nonredundant information were accepted as long as the agent, verb, and some of the additional information were specified. Furthermore, for cued-recall subjects, sentences not correctly paired with the experimental cues were unacceptable.

General criteria for acceptability were studied by two judges, who subsequently worked independently to provide a recall score for each subject. Interrater reliability coefficients for the recall scores of the two judges ranged from .93 to .97 for the four encoding $x$ retrieval conditions. Scoring discrepancies were resolved to provide a single set of scores for analysis.

\section{Results and Discussion}

Means and standard deviations for sentence recall are shown in Table 1. A two-factor analysis of variance indi-
Table 1

Sentence Recall in Experiment 1 as a Function of Encoding and Test Conditions

\begin{tabular}{|c|c|c|c|c|c|c|}
\hline \multirow{3}{*}{$\begin{array}{l}\text { Encoding } \\
\text { Condition }\end{array}$} & \multicolumn{6}{|c|}{ Test Condition } \\
\hline & \multicolumn{3}{|c|}{ Free Recall } & \multicolumn{3}{|c|}{ Cued Recall } \\
\hline & Mean & SD & $\mathrm{N}$ & Mean & SD & $\mathrm{N}$ \\
\hline Memorization & 6.47 & 2.28 & 19 & 6.65 & 2.89 & 20 \\
\hline Comprehension & 4.90 & 2.58 & 19 & 9.18 & 3.06 & 22 \\
\hline
\end{tabular}

cated a main effect of test $[\mathrm{F}(1,76)=14.0, \mathrm{p}<.001]$, but no main effect of encoding $(F<1)$. In general, cued recall was greater than free recall. There was a significant encoding $x$ test interaction $[F(1,76)=11.8, p<.001]$, suggesting that the encoding task had opposite effects in the two recall test conditions. Thus, tests of simple main effects were carried out. In cued recall, comprehension subjects outperformed the memorization subjects $[F(1,76)=8.98, p<.01]$. In free recall, however, there was a marginally significant tendency for comprehension subjects to recall less than memorizers $[F(1,76)$ $=3.49, p<.071$. Furthermore, it can be seen that comprehension subjects recalled more in cued recall than in free recall $[F(1,76)=15.2, p<.001]$. Type of test made no difference for memorization subjects $(F<1)$.

These results partially support the hypothesis that encoding/retrieval similarity contributes to good recall. Of the two encoding tasks, memorization provided the better preparation for free-recall testing, whereas the comprehension task provided the better preparation for cued-recall testing. In addition, however, the main effect of test may indicate that cued recall provides more distinctive retrieval aids than does free recall, for all subjects.

From another perspective, it appears that memorizers organize a set of sentences in memory and then attempt to reproduce them during recall, either by listing them spontaneously (free recall) or by listing them alongside seemingly appropriate cue words (cued recall). Additional data from a recent pilot study show that the performance of memorizers is relatively poor, compared with that of comprehension subjects, when cued recall is given subsequent to the 4-min free-recall period; very few additional sentences are cued. In contrast, comprehension subjects in the present study show greater memory in cued recall than in free recall, despite the apparently "deep" semantic encoding at input.

\section{EXPERIMENT 2}

Experiment 2 sought to replicate the study while examining two different presentation rates. Presumably, memorizers would benefit more from a slower presentation rate because of additional rehearsal activities performed in an effort to learn the material. Comprehension subjects, in contrast, would have no specific use for additional processing time. Again, the encoding $x$ test interaction was predicted, although the slower pre- 
sentation rate might improve the performance of all memorization subjects.

\section{Method}

Subjects. The subjects of Experiment 2 were drawn from the same population as that used in Experiment 1 and were given similar credit for participation. The subjects were tested in small groups assigned randomly to one of the presentation-rate (20 vs. $30 \mathrm{sec}$ ) $\mathrm{x}$ test (free recall vs. cued recall) conditions. Again, some subjects within a group were assigned the memorization task and others were assigned the comprehension task. There were 78 subjects in the $2 \times 2 \times 2$ design.

Materials. The stimulus materials and cue words were the same as those in Experiment 1.

Procedure. As in Experiment 1, the subjects in each session were arbitrarily assigned either the memorization task or the comprehension task. Instructions and scoring were the same as in the earlier experiment, except that the subjects were told they would have approximately 20 or $30 \mathrm{sec}$ between each stimulus sentence. Interrater reliability coefficients for the recall scores of the two judges ranged from .87 to .99 for the eight encoding $\mathrm{x}$ test $\mathrm{x}$ presentation-rate conditions.

\section{Results and Discussion}

Means and standard deviations for sentence recall are presented in Table 2. A three-factor analysis of variance indicated a main effect of presentation rate $[F(1,70)=$ $8.67, \mathrm{p}<.005]$. Recall was higher in the 30 -sec condition than in the 20 -sec condition. There were no significant interactions with presentation rate, although the means suggest that the slower presentation rate improved recall everywhere but in the comprehension/ free-recall condition.

The only other significant effect was the encoding $\mathrm{x}$ test interaction $[F(1,70)=5.96, p<.02]$. As in Experiment 1 , the encoding task appeared to have opposite effects in the two recall test conditions. Tests of simple main effects showed that in cued-recall comprehension subjects outperformed memorizers $[\mathrm{F}(1,70)=5.38$, $\mathrm{p}<.025$ ], but that in free recall there was no reliable encoding task effect $(F<1)$. Analyzing simple main effects on the other variable, we see that comprehension subjects performed much better in cued recall than in free recall $[F(1,70=5.77, p<.025]$. However, mem-

Table 2

Sentence Recall in Experiment 2 as a Function of Encoding, Test, and Presentation-Rate Conditions

\begin{tabular}{|c|c|c|c|c|c|c|}
\hline \multirow{3}{*}{$\begin{array}{c}\text { Encoding and } \\
\text { Presentation-Rate } \\
\text { Conditions }\end{array}$} & \multicolumn{6}{|c|}{ Test Condition } \\
\hline & \multicolumn{3}{|c|}{ Free Recall } & \multicolumn{3}{|c|}{ Cued Recall } \\
\hline & Mean & SD & $\mathrm{N}$ & Mean & SD & $\mathrm{N}$ \\
\hline & \multicolumn{6}{|c|}{ Memorization } \\
\hline $\begin{array}{l}20 \text {-sec Rate } \\
30 \text {-sec Rate } \\
\text { Both Rates }\end{array}$ & $\begin{array}{l}6.82 \\
8.33 \\
7.58^{*}\end{array}$ & $\begin{array}{l}1.54 \\
2.31\end{array}$ & $\begin{array}{r}11 \\
9\end{array}$ & $\begin{array}{l}5.12 \\
8.64 \\
6.88^{*}\end{array}$ & $\begin{array}{l}4.22 \\
3.19\end{array}$ & $\begin{array}{r}8 \\
11\end{array}$ \\
\hline & \multicolumn{6}{|c|}{ Comprehension } \\
\hline $\begin{array}{l}20 \text {-sec Rate } \\
30-\sec \text { Rate } \\
\text { Both Rates }\end{array}$ & $\begin{array}{l}6.73 \\
6.89 \\
6.81^{*}\end{array}$ & $\begin{array}{l}1.99 \\
2.45\end{array}$ & $\begin{array}{r}11 \\
9\end{array}$ & $\begin{array}{l}8.12 \\
9.73 \\
8.93^{*}\end{array}$ & $\begin{array}{l}2.09 \\
2.62\end{array}$ & $\begin{array}{r}8 \\
11\end{array}$ \\
\hline
\end{tabular}

*Unweighted means. orizers appeared to perform equally well in both recall test conditions $(\mathrm{F}<1)$.

Despite some differences between the two experiments, there was again partial support for the hypothesis that encoding/retrieval similarity facilitates recall. Although the comprehension/cued-recall condition produced better memory performance than the other conditions with which it was compared, the same cannot be said about the memorization/free-recall condition. As suggested in Experiment 1, it may be that comprehension/ cued-recall subjects benefit from the distinctiveness of the comprehension encoding, the distinctiveness of the implicational cues, and the similarity of these encoding and retrieval processes. In contrast, memorization/ free-recall subjects experience encoding/retrieval similarity without distinctive processing at input or test.

The main effect of presentation rate indicates that the additional processing time allowed both memorization and comprehension subjects to do more processing of the sentence stimuli. Apparently, all subjects continued to actively process sentences, even beyond the first $20 \mathrm{sec}$. Thus, recall improved in all conditions.

\section{GENERAL DISCUSSION}

Both experiments found some support for the hypothesis that encoding/retrieval similarity facilitates recall. Performance in the comprehension/cued-recall condition was consistently better than in the dissimilar-encoding/retrieval conditions (memorization/cued recall and comprehension/free recall). On the other hand, performance in the memorization/freerecall condition was not consistently better than it was in the dissimilar-encoding/retrieval conditions, and was often no different statistically. Thus, in the present study, the advantage of encoding/retrieval similarity for memory is clear only in the case of distinctive encoding (specific comprehension response to each sentence) and distinctive retrieval cues (implications as cues). As Tulving (1979) has suggested, variations in distinctiveness of encoding operations may be responsible for the fact that encoding/retrieval similarity leads to memory that is better in some cases than in others. This would mean, in the present study, that memorization produces a less distinctive encoding than does the comprehension task-a plausible notion, since memorization may include rehearsal of previous items already rehearsed on earlier trials. And, of course, free recall provides fewer distinctive cues than does cued recall.

\section{REFERENCES}

Anderson, R. C., \& Ortony, A. On putting apples into bottlesA problem of polysemy. Cognitive Psychology, 1975, 7, 167-180.

Corbett, A. T., \& Dosher, B. A. Instrument inferences in sentence encoding. Journal of Verbal Learning and Verbal Behavior, 1978, 17, 479-491.

Craik, F. I. M. Levels of processing: Overview and closing comments. In L. S. Cermak \& F. I. M. Craik (Eds.), Levels of processing in human memory. Hillsdale, N.J: Erlbaum, 1979.

JENkins, J. J. Four points to remember: A tetrahedral model of memory experiments. In L. S. Cermak \& F. I. M. Craik (Eds.), Levels of processing in human memory. Hillsdale, N.J: Erlbaum, 1979.

Morris, C. D., Bransford, J. D., \& Franks, J. J. Levels of processing versus transfer appropriate processing. Journal of Verbal Learning and Verbal Behavior, 1977, 16, 519-533.

Nelson, D. L. Remembering pictures and words: Appearance, 
significance, and name. In L. S. Cermak \& F. I. M. Craik (Eds.), Levels of processing in human memory. Hillsdale, N.J: Erlbaum, 1979.

TILL, R. E. Sentence memory prompted with inferential recall cues. Journal of Experimental Psychology: Human Learning and Memory, 1977, 3, 129-141.

Till, R. E., Cormak, D. R., \& Prince, P. L. Effects of orient- ing tasks on sentence comprehension and cued recall. Memory \& Cognition, 1977, 5, 59-66.

Tulving, E. Relation between encoding specificity and levels of processing. In L. S. Cermak \& F. I. M. Craik (Eds.), Levels of processing in human memory. Hillsdale, N. J: Erlbaum, 1979.

(Manuscript received for publication April 7, 1983.) 